\title{
IN SILICO MOLECULAR MODELING AND DOCKING OF APIGENIN AGAINST THE LUNG CANCER CELL PROTEINS
}

\author{
TULASI KASILINGAM ${ }^{1}$, ASITA ELENGOE ${ }^{2 *}$ \\ ${ }^{1}$ Department of Science and Biotechnology, Faculty of Engineering and Life Sciences, Universiti Selangor, 45600 Bestari Jaya, Selangor, \\ Malaysia. ${ }^{2}$ Department of Biotechnology, Faculty of Science, Lincoln University College, 47301 Petaling Jaya, Selangor, Malaysia. \\ Email: asitaelengoe@yahoo.com \\ Received: 11 April 2018, Revised and Accepted: 25 May 2018
}

ABSTRACT

Objective: In this study, three-dimensional (3D) structures of lung cancer cell line proteins (cellular tumor antigen [p53], caspase 3, and mucosal addressin cell adhesion molecule 1) were generated, and their binding affinities with apigenin through local docking were studied.

Methods: The lung cancer cell line proteins were built using Swiss model and visualized by the PyMol software. The physicochemical characterization of the protein models was evaluated by Expasy's ProtParam Proteomics server. Then, they were validated by PROCHECK, ProQ, ERRAT, and Verify 3D programs. Finally, the protein models were docked with apigenin using BSP-Slim server.

Results: All the protein models were acceptable and of good quality. The apigenin showed the binding energy with cellular tumor antigen (p53), caspase 3 , and mucosal addressin cell adhesion molecule 1 at $-4.611,-5.750$, and $-5.307 \mathrm{kcal} / \mathrm{mol}$, respectively.

Conclusion: The caspase 3 had the strongest bond with apigenin. These potential drug candidates can further be validated in laboratory experiments for its proper function.

Keywords: Cellular tumor antigen (p53), Caspase 3, Mucosal addressin cell adhesion molecule 1, Apigenin, Docking.

(C) 2018 The Authors. Published by Innovare Academic Sciences Pvt Ltd. This is an open access article under the CC BY license (http://creativecommons. org/licenses/by/4. 0/) DOI: http://dx.doi.org/10.22159/ajpcr.2018.v11i9.26649

\section{INTRODUCTION}

Lung cancer is the leading cause of death in Malaysia. In 2007, it is the third most common cancer after breast and colorectal cancer. It is the most common cancer diagnosed in men while fifth common cancer in women [1]. In 2006, 2948 lung cancer cases were reported (1445 in men and 603 in women). Moreover, 1.8 million lung cancer cases were estimated to have been diagnosed in 2012 .

Even though the survival rate after a diagnosis of lung cancer made is $49 \%$; only $<2 \%$ of cancer patients are still alive in 5 years if cancer has not spread to the other parts of the body. The incidence of lung cancer and consequent death from this disease is expected to increase over the next decade because of the high rates of smoking [1].

The mean age at which lung cancer is diagnosed in Malaysia is about 60 years with a peak age of diagnosis in the $7^{\text {th }}$ decade. The incidence of diagnosed lung cancer in Malaysian patients aged $<40$ years is generally low at about $6.2 \%$. Based on the World Health Organization (WHO) performance status, younger patients were more likely than older patients to have adenocarcinoma with poorer. Late stage introduction and in this way inoperability is extremely normal in the more youthful age bunches as they more often than not stay asymptomatic or ignore symptoms longer [2]. In one study, all patients $<40$ years old with non-small cell lung cancer (NSCLC) presented with either Stage IIIb or metastatic disease, compared to $77 \%$ of older patients [3].

Lung cancer usually divided into 2 groups according to its type of cells. First is NSCLC which can be subdivided into three, namely adenocarcinoma (30-40\%), squamous cell carcinoma (30\%), and large cell carcinoma (10\%). Second is small cell lung cancer $(20 \%)$ which cancer cell grows at a very rapid rate and most of the time spread to other parts of the diagnosis. Other types of cancer can be found in the lungs are carcinoid and lymphoma (5\%) [4]. Thus, the development of a therapeutic approach to lung cancer remains one of the most challenging areas in cancer research.

Plant compounds also have been identified as a class of promising anticancer agents. Apigenin, plant compound found in parsley, has over and again been found to have solid against malignancy properties. Truth be told, more than 600 PubMed-recorded diary articles identify with the compound's part in the disease. In a look into distributed September 2015 in Oncotarget, researchers interestingly could recognize how apigenin can successfully back off or stop an undesirable compound, which assumes a part in disease movement.

Apigenin has increased specific interest for many years as a valuable and well-being promoting agent due to its low inherent poisonous quality and in light of its striking impacts on normal versus tumor cells, contrasted and other fundamentally related flavonoids [5]. There is almost no confirmation to date to recommend that apigenin helps adverse metabolic responses in vivo when expended as a component of a normal eating regimen. Apigenin has been progressively perceived in the recent years as a tumor chemopreventive agent. A study shows where the consumption of free flavonoids in healthy human volunteers diets lead to decrease in markers of oxidative stress in blood, namely plasma antioxidant vitamins, erythrocyte superoxide dismutase activity, and lymphocyte DNA damage commonly associated with enhanced disease risk [6].

In mammalian systems either in vitro or in vivo, a few of the biological property of apigenin are identified such as antioxidant effects and its part scavenging free radicals. Besides that, it exhibits antimutagenic, anti-inflammatory, antiviral, and purgative impacts [7]. The activities of apigenin in inhibiting the cell cycle, reducing oxidative stress, enhancing the viability of detoxification enzymes, inducing apoptosis, and stimulating the immune system are quite limited. 
Johnson et al. study demonstrated that apigenin had the inhibitory effects on glycogen synthase kinase (GSK-3ß) [8]. Inhibition of GSK$3 \beta$ causes decreased in the proliferation of pancreatic cancer cell and survival by abrogating nuclear factor $\kappa \mathrm{B}$ activity [8]. Apigenin docked with GSK-3 $\beta$. The binding energy of the apigenin - GSK$3 \beta$ complex was $76.1 \mathrm{kcal} / \mathrm{mol}$. Furthermore, Way et al. study described that apigenin docked with PI3K [9]. Therefore, PI3K could not bind with HER2/HER3 heterodimer which causes breast cancer proliferation. Apigenin indirectly inhibited PI3K activity in AKT pathway, but the interaction mechanism between cancer cell line proteins and apigenin is yet to be elucidated. In this study, we report for the first time that the cellular tumor antigen $\mathrm{p} 53$, caspase 3 , and mucosal addressin cell adhesion molecule 1 interacts with apigenin.

The objective of this study is to generate three-dimensional (3D) structures of lung cancer cell line proteins (cellular tumor antigen p53, caspase 3, and mucosal addressin cell adhesion molecule 1) and to evaluate their binding affinities with apigenin through local docking.

\section{METHODS}

\section{Target sequence}

The complete amino acid sequence of the p53 (GI: 2491729), caspase 3 (GI: 16516817), and mucosal addressin cell adhesion molecule 1 (GI: 109633022) were obtained from National Center for Biotechnology Information. It consists of 393, 277, and 382 amino acids, respectively.

\section{Homology modeling}

To date, the 3D models of cellular tumor antigen p53, caspase 3 , and mucosal addressin cell adhesion molecule 1 were not available. Hence, the models were generated using Swiss-Model (https://swissmodel. expasy.org/) [10] and visualized by the PyMol software (http://www. pymol.org// [11].

\section{Physiochemical characterization}

Protein structural analysis was carried out through the Expasy's ProtParam Proteomics server [12]. Non-polar and polar residues were determined by Color Protein Sequence (Colorseq) analysis [13]. The ESBRI program [14] was used to discover salt bridges in the protein models; the number of disulfide bonds was calculated using the Cys_Rec program [15].

\section{Prediction of the secondary structures}

The secondary structural features were predicted with Self-Optimized Prediction Method from Alignment (SOPMA) [16].

\section{Evaluation of the protein models}

The protein structures were validated with PROCHECK by Ramachandran plot analysis [17]. The model was further analyzed by ProQ [18], ERRAT [19], and verify 3D [20] programs.

\section{Identification of active sites}

To identify the binding sites of the cellular tumor antigen p53, caspase 3 , and mucosal addressin cell adhesion molecule 1 , they were submitted to active site-prediction server [21].
Preparation of ligand model

The tertiary structure of the apigenin was not publicly available. The complete sequence of apigenin obtained from PubChem (https:// pubchem.ncbi.nlm.nih.gov/) [22]. Then, the structure was in structured data format. Thus, it was converted to protein data bank format using molecule file format conversion (through Chem Axon J Chem).

\section{Protein-ligand docking}

The 3D model of cellular tumor antigen p53 was performed for molecular docking with the 3D structure of apigenin using BSP-Slim server [23]. The top-ranked conformation with the lowest binding energy was selected [24]. The same docking simulation approach was performed with the other two protein molecules.

\section{RESULTS AND DISCUSSION}

\section{Physiochemical characterization}

The computed isoelectric point (pI) value for cellular tumor antigen p53, caspase 3, and mucosal addressin cell adhesion molecule 1 $(\mathrm{pI}<7)$ indicated acidic characteristic. Moreover, p53, caspase 3, and mucosal addressin cell adhesion molecule 1 had a molecular weight of 43683.16, 31641.92, and 40155.28 Daltons, respectively. Through the extent of light that is absorbed by a protein, the elimination coefficient of tyrosine, cysteine, and tryptophan residues was calculated at a specific wavelength, which is $31400-35410 \mathrm{M} / \mathrm{cm}$. Moreover, ASP+GLU is the total number of negatively charged residues which are $-R$, and $\mathrm{ARG}+\mathrm{LYS}$ is $+\mathrm{R}$ which is the total number of positively charged residues. Thus, there were was a sum of 50 negatively charged residues and 46 positively charged residues for cellular tumor antigen p53 in the amino acid sequence, while caspase 3 had a total of 40 negatively charged residues and 36 positively charged residues in the amino acid sequence. Besides that, mucosal addressin cell adhesion molecule 1 had a sum of 40 positively charged residues and 26 negatively charged residues in the amino acid sequence. Based on the Expasy's ProtParam instability index, cellular tumor protein p53 and mucosal addressin cell adhesion molecule 1 proteins were classified as unstable because the instability index value was more than 40 which had an instability index value of 71.93 and 63.75 . However, caspase 3 can be classified as stable as it had stability index within the range.

The aliphatic list of all the protein models is shown in Table 1 . The low grand average of hydropathicity (GRAVY) index (a negative esteem GRAVY) of all proteins is exhibited their hydrophilic nature (Table 1). Furthermore, cellular tumor antigen p53, caspase 3, and mucosal addressin cell adhesion molecule 1 had more polar residues $(47.33,49.10$, and $41.36 \%)$ than nonpolar residues $(24.94,27.44$, and $31.94 \%$ ). This was determined using Color Protein Sequence analysis.

Salt bridges have an important role in the structure and stability of a protein. The interruption of a salt bridge reduces the protein stability [25]. It is additionally engaged with allosteric control, recognition of molecular, oligomerization, adaptability, area movements, and thermostability $[26,27]$. In this study, the cellular tumor antigen p53, caspase 3 , and mucosal addressin cell adhesion molecule 1 had 6 , 6 , and 6 salt bridges, respectively, which were obtained through ESBRI. They were formed by residues of arginine.

Table 1: Physiochemical characters of the cellular tumor antigen p53, caspase 3, and mucosal addressin cell adhesion molecule 1 as predicted by Expasy's protParam program

\begin{tabular}{|c|c|c|c|c|c|c|c|c|c|}
\hline Protein & Length & $\begin{array}{l}\text { Molecular } \\
\text { weight }\end{array}$ & pI & $-\mathbf{R}$ & $+\mathbf{R}$ & $\begin{array}{l}\text { Extraction } \\
\text { coefficient }\end{array}$ & $\begin{array}{l}\text { Instability } \\
\text { index }\end{array}$ & $\begin{array}{l}\text { Aliphatic } \\
\text { index }\end{array}$ & GRAVY \\
\hline Cellular tumor antigen p53 & 393 & 43683.16 & 6.37 & 50 & 46 & 35410 & 71.93 & 59.08 & -0.758 \\
\hline Caspase 3 & 277 & 31641.92 & 6.09 & 40 & 36 & 31400 & 40.58 & 70.04 & -0.472 \\
\hline $\begin{array}{l}\text { Mucosal addressin cell } \\
\text { adhesion molecule } 1\end{array}$ & 382 & 40155.28 & 5.00 & 40 & 26 & 31970 & 63.75 & 84.27 & -0.250 \\
\hline
\end{tabular}

GRAVY: Grand average of hydropathicity 
The thermostability of a molecule is increased in the presence of arginine in the protein structure by giving more electrostatic interactions through their guanidine group [28]. Therefore, it ensured that all the proteins are in the same stability. From the analysis of Cys_Rec, it shows that the number of disulfide bonds in cellular tumor antigen $\mathrm{p} 53$, caspase 3 , and mucosal addressin cell adhesion molecule 1 had 10,8 , and 7 , respectively (Table 2 ).

Table 2: Presence of disulfide (ss) bond predicted by Cys_Rec server

\begin{tabular}{lll}
\hline Protein & Cys_Rec & Score \\
\hline Cellular tumor antigen p53 & Cys_124 & -10.0 \\
& Cys_135 & 0.3 \\
& Cys_141 & -9.7 \\
& Cys_176 & -12.1 \\
& Cy__182 & -46.5 \\
& Cys_229 & -55.4 \\
& Cys_238 & -1.2 \\
& Cys_242 & -9.5 \\
& Cys_275 & -35.1 \\
Caspase 3 & Cys_277 & -50.8 \\
& Cys47 & -67.8 \\
& Cys_116 & -60.0 \\
& Cys_148 & -66.7 \\
& Cys_163 & -68.7 \\
Cys_170 & -44.6 \\
adhesion molecule 1 & Cy_184 & -57.9 \\
& Cys_220 & -58.3 \\
& Cys_264 & -59.4 \\
& Cys_47 & 56.0 \\
& & \\
& Cys_51 & 61.7 \\
& Cys_94 & 50.9 \\
& Cys_98 & 46.7 \\
& Cys_134 & 24.7 \\
& Cys_204 & 42.1 \\
& Cys_346 & -21.6 \\
\hline
\end{tabular}

Prediction of the secondary structures

SOPMA view has demonstrated that the presence of random coils overwhelmed among secondary structure components took after by $\alpha$-helix, broadened strand, and beta turns at different positions in proteins. The composition of $\alpha$-helix in cellular tumor antigen p53, caspase 3, and mucosal addressin cell adhesion molecule 1 was shown in Table 3.

Results from this analysis indicated that cellular tumor antigen p53, caspase 3 , and mucosal addressin cell adhesion molecule 1 consist of 11,12 , and $15 \alpha$ helices, respectively. The longest and shortest $\alpha$ helix of all proteins is described in Table 4.

\section{Validation of the protein models}

For the stereochemical quality and the geometry of protein models, they were validated by Ramachandran plot calculations through PROCHECK software (Fig. 1). From the analysis, it was confirmed that residues of all protein models are in a most favorable region which was more than $80 \%$ (Table 5). Therefore, the quality of these proteins was evaluated to be great and dependable. PROCHECK analysis revealed that several residues such as ARG76, LEU194, ASP129, SER66, ASP64, ASP73, ASN145, and SER229 were situated out of energetically favored regions of Ramachandran plot for mucosal addressin cell adhesion molecule 1. However, there were no residues found at the disallowed region for other two protein models.

Hence, the stereochemical evaluation of backbone phi/psi dihedral angles inferred that a low percentage of residues for cellular tumor antigen p53, caspase 3 , and mucosal addressin cell adhesion molecule 1 were falling within protein models. ProQ was used to verify "the quality" using the Levitt-Gerstein (LG) score and maximum subarray (MaxSub). The results show that the predicted LG score ( $>1.5$ : Good model) and predicted MaxSub score ( $>0.1$ good model) for all protein structures were in the acceptable range to create a good model (Table 5).

ERRAT is an analysis for evaluating protein models determined by X-ray crystallography. ERRAT value depends on the statistics of nonbonded atomic interactions in the 3D protein structure. The normally accepted

Table 3: Secondary structure of the cellular tumor antigen p53, caspase 3, and mucosal addressin cell adhesion molecule 1

\begin{tabular}{llll}
\hline Secondary structure & $\alpha$ helix & Extended strand & Beta-turn \\
\hline Cellular tumor antigen p53 & 18.83 & 19.08 & 8.91 \\
Caspase 3 & 35.02 & 17.33 & 9.75 \\
Mucosal addressin cell adhesion molecule 1 & 28.27 & 14.40 & 37.92 \\
\hline
\end{tabular}

Table 4: Composition of $\alpha$-helix in the cellular tumor antigen p53, caspase 3, and mucosal addressin cell adhesion molecule 1

\begin{tabular}{llll}
\hline Amino acid & Longest $\boldsymbol{\alpha}$-helix & Residues & Shortest $\alpha$-helix \\
\hline Cellular tumor antigen p53 & $\alpha_{9}$ & 16 & $\alpha_{8}$ \\
Caspase 3 & $\alpha_{9}$ & 36 & $\alpha_{5}, \alpha_{8}$ \\
Mucosal addressin cell adhesion molecule 1 & $\alpha_{13}$ & 1 & $\alpha_{2}, \alpha_{4}$ \\
\hline
\end{tabular}

Table 5: Validation of the cellular tumor antigen p53, caspase 3, and mucosal addressin cell adhesion molecule 1

\begin{tabular}{|c|c|c|c|c|c|c|c|c|c|}
\hline \multirow[t]{2}{*}{ Structure } & \multicolumn{4}{|c|}{ Ramachandran plot statistics (\%) } & \multicolumn{3}{|c|}{ Goodness factor } & \multicolumn{2}{|l|}{ ProQ } \\
\hline & $\begin{array}{l}\text { Most } \\
\text { favored }\end{array}$ & $\begin{array}{l}\text { Additionally } \\
\text { allowed }\end{array}$ & $\begin{array}{l}\text { Generously } \\
\text { allowed }\end{array}$ & Disallowed & $\begin{array}{l}\text { Dihedral } \\
\text { angles }\end{array}$ & $\begin{array}{l}\text { Overall } \\
\text { forces }\end{array}$ & $\begin{array}{l}\text { Overall } \\
\text { average }\end{array}$ & $\begin{array}{l}\text { LG } \\
\text { score }\end{array}$ & $\begin{array}{l}\text { Max } \\
\text { Sub }\end{array}$ \\
\hline p53 & 89.8 & 9.6 & 0.6 & 0.0 & -0.33 & -0.04 & -0.20 & 4.527 & 0.478 \\
\hline Caspase 3 & 92.5 & 7.0 & 0.4 & 0.0 & -0.14 & 0.04 & -0.06 & 2.193 & 0.191 \\
\hline $\begin{array}{l}\text { Mucosal } \\
\text { addressin } \\
\text { cell } \\
\text { adhesion } \\
\text { molecule } 1\end{array}$ & 82.2 & 10.7 & 5.3 & 1.8 & -0.68 & -0.39 & 0.53 & 4.212 & 0.348 \\
\hline
\end{tabular}




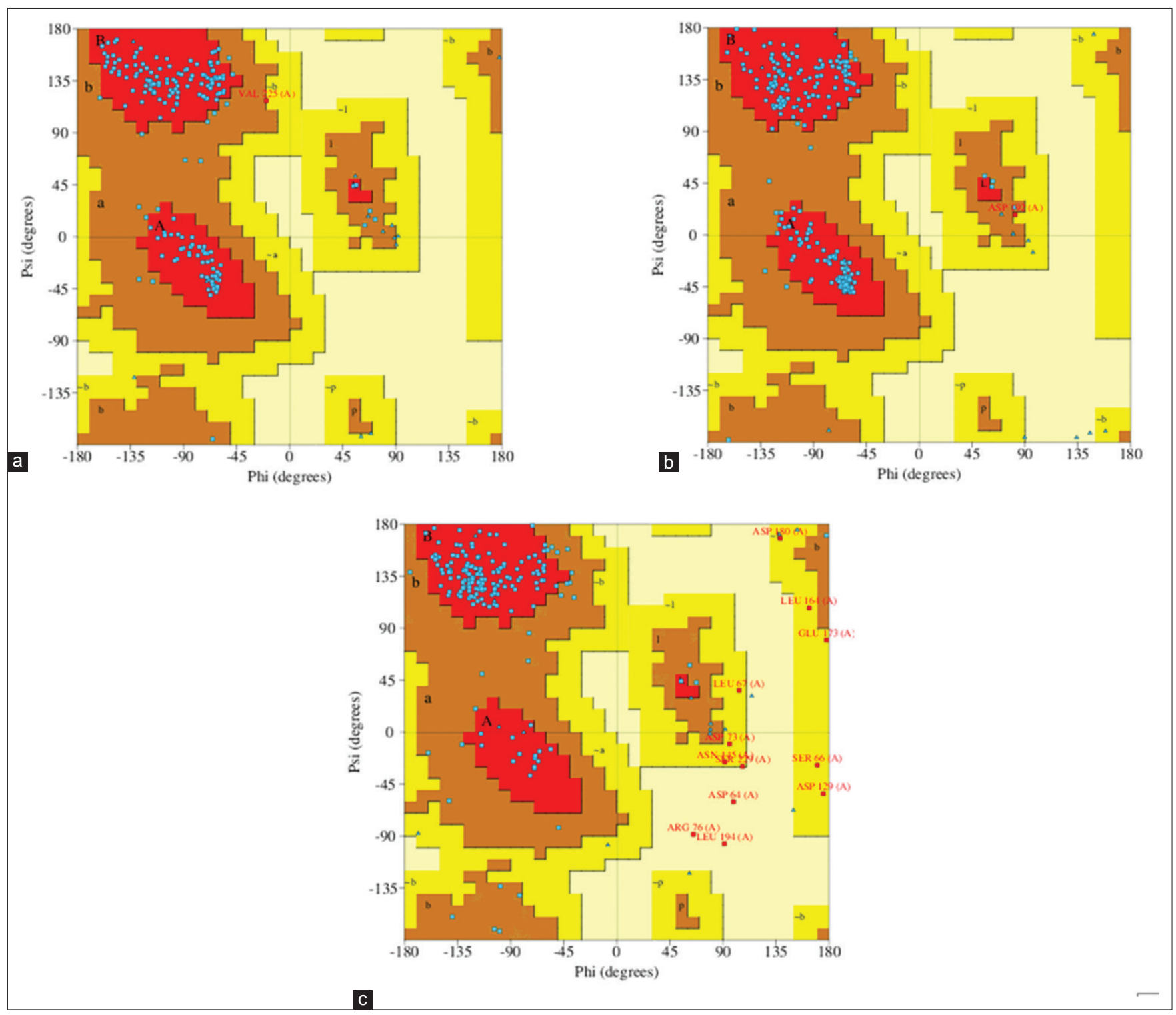

Fig. 1: Ramachandran plots for (a) cellular tumor antigen p53, (b) Caspase 3, and (c) mucosal addressin cell adhesion molecule 1

range is more than $50 \%$ for a high-quality protein model. The result analysis of ERRAT score revealed that caspase 3 was the highest at 98.492 (Fig. 2). Thus, it is confirmed that caspase 3 model has a good high resolution and quality model compared to other protein models. The scores for the cellular tumor antigen p53 and mucosal addressin cell adhesion molecule 1 were 93.514 and 86.928 , respectively.

Cellular tumor antigen p53, caspase 3, and mucosal addressin cell adhesion molecule 1 had $85.71 \%, 71.49 \%$, and $95.69 \%$ of the residues, respectively, which were revealed through verifying with the 3D server. It had an average 3D-1D score of more than 0.2. Hence, it indicates all the sequence were compatible with its protein model (Fig. 3). Finally, the resulting energy minimized cellular tumor antigen p53, caspase 3 , and mucosal addressin cell adhesion molecule 1 protein models fulfilled the criteria for the protein evaluation. Thus, docking analysis with ligand will proceed.

\section{Identification of active sites}

The protein volume of the active site and the residues forming a pocket of the active site for cellular tumor antigen p53, caspase 3 , and molecular addressin cell adhesion molecule 1 were obtained using BSP Slim server (Table 6). The protein volume for cellular tumor antigen p53, caspase 3, and mucosal addressin cell adhesion molecule 1 is 1229 , 1164 , and $938 \mathrm{~A}^{3}$, respectively.

\section{Protein-ligand docking}

Two early human papillomavirus (HPV) genes, E6 and E7, are known to play crucial role in tumor formation. E6 binds with p53 and prevents its translocation and thereby inhibit the ability of p53 to activate or repress target genes. $\mathrm{E} 7$ binds to hypophosphorylated $\mathrm{Rb}$ and thereby induces cells to enter into premature S-phase by disrupting Rb-E2F complexes. The aim of the research work was to target the site of interaction of Rb1-E7 and p53-E6. A total of 88 compounds were selected for molecular screening, based on comprehensive literature survey for natural compounds with anticancer activity. Molecular docking analysis was carried out with Molegro Virtual Docker to study on their binding affinity toward the site of interaction of the viral oncoproteins and human tumor suppressor proteins. The docking analysis revealed that Nicandrenone a member of Withanolides family of chemical compounds as the most likely molecule that can be used as a candidate drug against HPV induced cervical cancer [29].

In this study, cellular tumor antigen p53, caspase 3 , and mucosal addressin cell molecule 1 were successfully docked with apigenin using the BSP- 


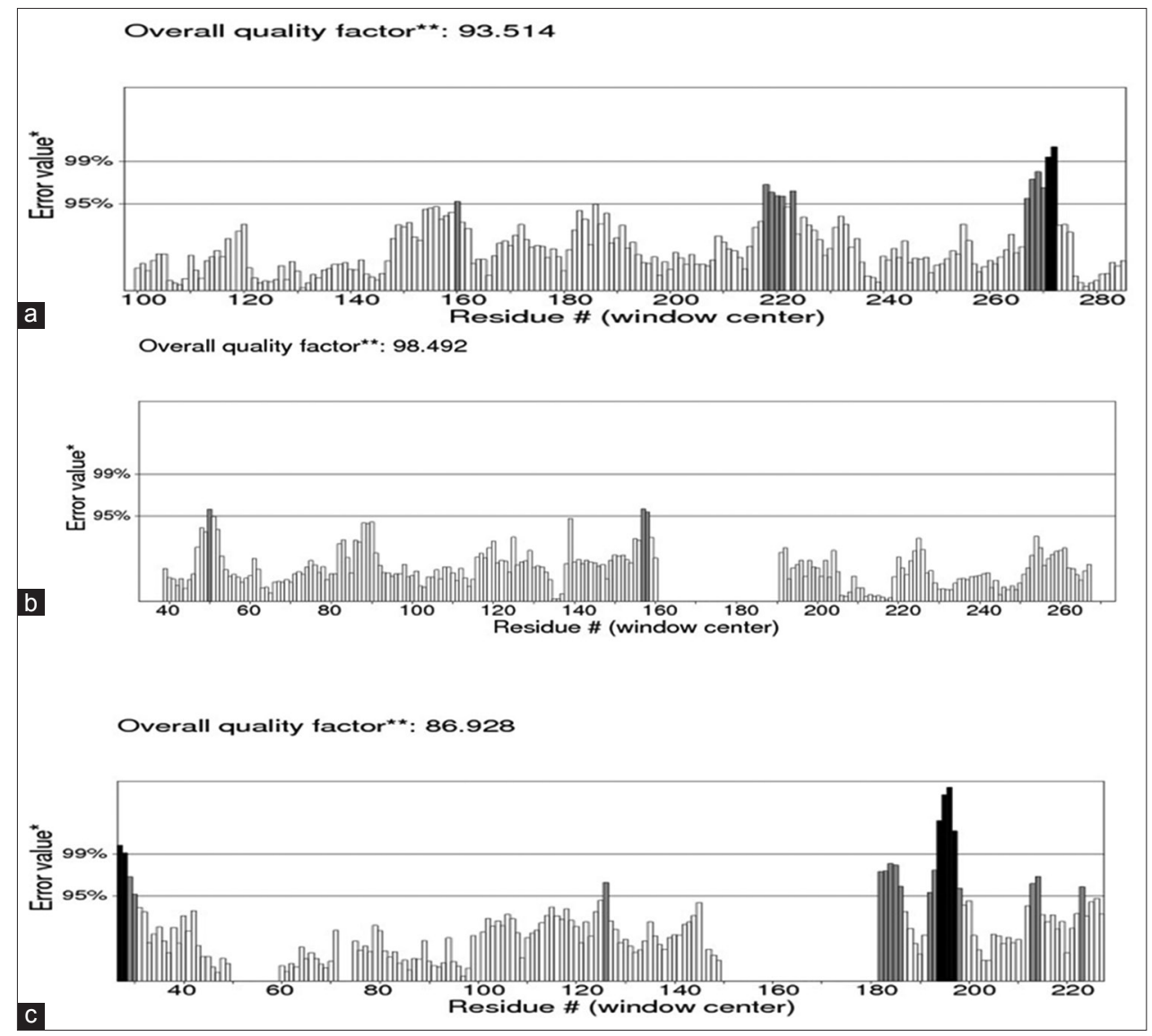

Fig. 2: ERRAT plots for (a) cellular tumor antigen p53, (b) caspase 3, and (c) mucosal addressin cell adhesion molecule 1

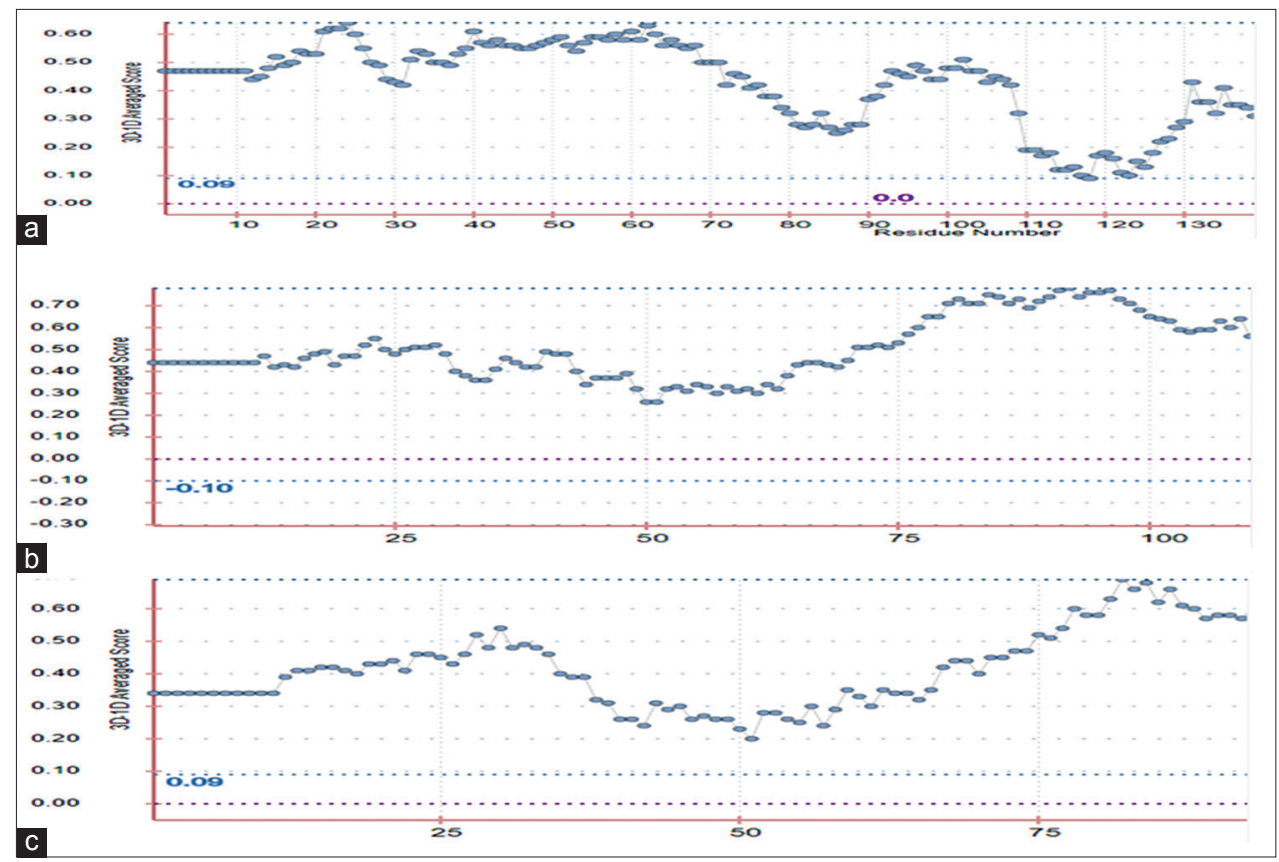

Fig. 3: Verify three-dimensional plots for (a) cellular tumor antigen p53, (b) caspase 3, and (c) mucosal addressin cell adhesion molecule

Slim server. Based on the negative and lowest energy value ( $\mathrm{D}_{G}$ bind), the best docking orientation was chosen [30,31]. Docking analysis showed that there was a strong interaction between cellular tumor antigen p53 and apigenin. It was the most favorable because it had the lowest energy value $(-4.611 \mathrm{kcal} / \mathrm{mol})$ when compared to caspase 3 and mucosal addressin cell adhesion molecule 1 which had -5.750 and $-5.307 \mathrm{kcal} /$ mol, respectively (Table 7). The binding energy was calculated as the sum of the intermolecular energy and torsion energy. 
Table 6: Predicted active sites of the cellular tumor antigen p53, caspase 3, and mucosal addressin cell adhesion molecule 1

\begin{tabular}{|c|c|c|}
\hline Protein & Volume & Residues that forming pocket \\
\hline Cellular tumor antigen p53 & 1229 & $\begin{array}{l}\text { TYR10, GLN11, GLY12, ASP135, CYS136, THR138, TYR141, PR0157, LEU159, } \\
\text { ARG17, ASN175, SER176, GLU178, VAL179, LEU18, HIE180, ARG189, GLY19, } \\
\text { GLU192, GLU193, LEU196, PHE20, LEU21, HIE22, TYR33, PR035, ALA36, } \\
\text { LEU37, ASN38, LYS39, PR049, GLN51, TRP53, ASP55, GLN7, LYS71, LYS8, THR9 }\end{array}$ \\
\hline Caspase 3 & 1164 & $\begin{array}{l}\text { VAL106, ASP107, LEU108, LYS109, MET11, LYS110, ILE111, THR112, ASN113, } \\
\text { PHE114, PHE115, ARG116, GLY117, LYS126, PR0127, LYS128, LEU129, PHE130, } \\
\text { ILE131, ILE132, GLN133, ALA134, CYS135, ARG136, GLY137, ILE20, ASN24, } \\
\text { ARG36, THR39, ASP42, ASN45, LEU46, THR49, PHE50, LEU53, TYR55, MET72, } \\
\text { SER85, PHE86, VAL87, CYS88, VAL89, TYR9, LEU90, LEU91, SER92, GLY94, } \\
\text { GLU95, GLU96, GLY97, ILE98, ILE99 }\end{array}$ \\
\hline Mucosal addressin cell adhesion molecule 1 & 938 & $\begin{array}{l}\text { LYS116, THR118, PR0119, VAL12, VAL120, ASP121, PR0122, ASN123, ALA124, } \\
\text { LEU125, PHE127, PR0144, GLU145, VAL146, GLN147, GLU148, GLU149, } \\
\text { GLU150, GLU157, ASP158, VAL159, ALA16, LEU160, PHE161, VAL163, LEU17, } \\
\text { GLY18, ARG187, LEU188, PR0189, LEU41, THR43, LEU45, SER63, LEU64, } \\
\text { SER65, ALA66, ALA67, GLY68, THR69, ARG70, GLN86, LEU87, LEU88, VAL89, } \\
\text { TYR90, PHE92, PR093, ASP94 }\end{array}$ \\
\hline
\end{tabular}

Table 7: Docking result of the cellular tumor antigen p53, caspase 3, and mucosal addressin cell adhesion molecule 1

\begin{tabular}{llll}
\hline Protein & Cellular tumor antigen p53 & Caspase 3 & Mucosal addressin cell adhesion molecule 1 \\
\hline Binding energy $(\mathrm{kcal} / \mathrm{mol})$ & -4.611 & -5.750 & -5.307 \\
\hline
\end{tabular}

\section{CONCLUSION}

Cellular tumor antigen p53, caspase 3 , and mucosal addressin cell adhesion molecule 1 were successfully docked with apigenin. The three protein models had a strong interaction between apigenin due to their negative binding energy. Thus, advance biochemical and in vivo investigations in silico interpretations of this protein-ligand model will support in designing more a potent structure-based drug.

\section{AUTHOR'S CONTRIBUTIONS}

All the authors contributed equally.

\section{CONFLICTS OF INTEREST}

All authors of this publication declare that there are no conflicts of interest in publishing this research article.

\section{REFERENCES}

1. Brambt IE, Travis WD. Lung Cancer. World Cancer Report. World Health Organization; 2014. p. 489-508.

2. Koo LC, Ho JH. Worldwide epidemiological patterns of lung cancer in non-smokers. Int J Epidemiol 1990;19:S14-23.

3. Toh CK, Wong EH, Lim WT. The impact of smoking status on the behaviour and survival outcome of patients with advanced non-small cell lung cancer: A retrospective analysis. Chest 2004;126:1750-6.

4. Chong KL, Kim HL, Catherine MM. Lung cancer in patients younger than 40 years in a multiracial Asian country. Respiratory 2000;5:355-61.

5. Gupta S, Afaq F, Mukhtar H. Selective growth-inhibitory, cell-cycle deregulatory and apoptotic response of apigenin in normal versus human prostate carcinoma cells. Biochem Biophys Res Commun 2001;287:914-20.

6. Kim HY, Kim OH, Sung MK. Effects of phenol-depleted and phenolrich diets on blood markers of oxidative stress, and urinary excretion of quercetin and kaempferol in healthy volunteers. J Am Coll Nutr 2003;22:217-23.

7. Yang CS, Landau JM, Huang MT, Newmark HL. Inhibition of carcinogenesis by dietary polyphenolic compounds. Annu Rev Nutr 2001;21:381-406.

8. Johnson JL, Rupasinghe SG, Stefani F, Schuler MA, Gonzalez de Mejia, E. Citrus flavonoids luteolin, apigenin, and quercetin inhibit glycogen synthase kinase- $3 \beta$ enzymatic activity by lowering the interaction energy within the binding cavity. J Med Food 2011;14:325-33
9. Way TD, Kao MC, Lin JK. Apigenin induces apoptosis through proteasomal degradation of HER2/neu in HER2/neu-overexpressing breast cancer cells via the phosphatidylinositol 3-kinase/Akt-dependent pathway. J Biol Chem 2004;279:4479-89.

10. Biasini M, Bienert S, Waterhouse A, Arnold K, Studer G, Schmidt T, et al. SWISS-MODEL: Modelling protein tertiary and quaternary structure using evolutionary information. Nucleic Acids Res 2014;42:W252-8.

11. Delano WL. The PyMOL Molecular Graphics System. Palo Alto, CA: DeLano Scientific; 2001. Available from: http://www.pymol.org.

12. Gasteiger E, Hoogland C, Gattiker A, Duvaud S, Wilkins MR, Appel RD, et al. Protein identification and analysis tools on the ExPASy server. In: Walker JM, editor. The Proteomics Protocols Handbook. Totowa: Humana Press; 2005.

13. Prabi LG. Color Protein Sequence Analysis; 1998.Avaliable from: https://npsaprabi.ibcp.fr/cgi-bin/npsa_automat.pl?page=/NPSA/npsa_ color.html.

14. Costantini S, Colonna G, Facchiano AM. ESBRI: A web server for evaluating salt bridges in proteins. Bioinformation 2008;3:137-8.

15. Roy S, Maheshwari N, Chauhan R, Sen NK, Sharma A. Structure prediction and functional characterization of secondary metabolite proteins of Ocimum. Bioinformation 2011;6:315-9.

16. Geourjon C, Deleage G. SOPMA: Significant improvements in protein secondary structure prediction by consensus prediction from multiple alignments. Comput Appl Biosci 1995;11:681-4.

17. Laskowski RA, MacArthur MW, Moss DS, Thornton JM. PROCHECK: A program to check the stereo chemical quality of protein structures. J Appl Crystallogr 1993;26:283-91.

18. Wallner B, Elofsson A. Can correct protein models be identified? Protein Sci 2003;12:1073-86.

19. Colovos C, Yeates TO. Verification of protein structures: Patterns of non-bonded atomic interactions. Protein Sci 1993;2:1511-9.

20. Eisenberg D, Luthy R, Bowie JU. VERIFY3D: Assessment of protein models with three-dimensional profiles. Methods Enzymol 1977;277:396-404.

21. Jayaram B. Active Site Prediction server; 2004. Available from: http:// www.scfbio-iitd.res.in/dock/ActiveSite.jsp.

22. Kim S, Thiessen PA, Bolton EE, Chen J, Fu G, Gindulyte A, Han L, He J, He S, Shoemaker BA, Wang J, Yu B, Zhang J, Bryant SH. PubChem Substance and Compound databases. Nucleic Acids Res 2016;44:D1202-13.

23. Hui SL, Yang Z. BSP-SLIM: A blind low-resolution ligand-protein docking approach using theoretically predicted protein structures. Proteins 2012;80:93-110.

24. Purwanggana A, Mumpuni E, Mulatsari E. In vitro and in silico antibacterial activity of 1.5-Bis (3'-ethoxy-4-hydroxyphenyl)-1-4- 
Pentadiene-3-one. Int J Pharm Pharm Sci 2018;10:70-6.

25. Kumar S, Tsai CJ, Ma B, Nussinov, R. Contribution of salt bridges toward protein thermo stability. J Biomol Struct Dyn 2000;1:79-86.

26. Kumar S, Nussinov R. Salt bridge stability in monomeric proteins. J Mol Bio 2009;293:1241-55.

27. Kumar S, Nussinov R. Relationship between ion pair geometries and electrostatic strengths in proteins. Biophys J 2002;83:1595-612.

28. Parvizpour S, Shamsir MS, Razmara J, Ramli AN, Md Illias R. Structural and functional analysis of a novel psychrophilic b-mannanase from
Glaciozyma antarctica PI12. J Comput Aided Mol Des 2014;28:685-98.

29. Shaikh F, Sanehi P, Rawal R. Molecular screening of compounds to the predicted protein-protein interaction site of Rb1-E7 with p53- E6 in HPV. Bioinformation 2012;8:607-12.

30. Lourthuraji A, Masilamani S, Ravikrishnan B, Vinoth M, Hopper W. Analysis of molecular docking efficiency of Cleistanthin-A, as an alternative for nicotine addiction. Int J Pharm Pharm Sci 2018;10:98-100.

31. Barua $\mathrm{H}, \mathrm{Bhagat} \mathrm{N}$, Toraskar M. Study of binding interactions of human carbonic anhydrase XII. Int J Curr Pharm Res 2017;9:118-25. 